\title{
Observation of propane cluster size distributions during nucleation and growth in a Laval expansion
}

\section{Journal Article}

\section{Author(s):}

Ferreiro, Jorge J.; Chakrabarty, Satrajit; Schläppi, Bernhard; Signorell, Ruth (1)

Publication date:

2016-12

\section{Permanent link:}

https://doi.org/10.3929/ethz-b-000119287

Rights / license:

In Copyright - Non-Commercial Use Permitted

Originally published in:

The Journal of Chemical Physics 145(21), https://doi.org/10.1063/1.4960050

Funding acknowledgement:

159205 - Interaction of Light with Ultrafine Aerosol Particles II (SNF) 


\section{Observation of propane cluster size distributions during nucleation and growth in a Laval expansion}

Cite as: J. Chem. Phys. 145, 211907 (2016); https:// doi.org/10.1063/1.4960050

Submitted: 06 June 2016 . Accepted: 18 July 2016 . Published Online: 30 November 2016

Jorge J. Ferreiro, Satrajit Chakrabarty, Bernhard Schläppi, and Ruth Signorell

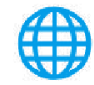

View Online

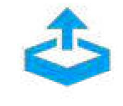

Export Citation

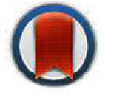

CrossMark

\section{ARTICLES YOU MAY BE INTERESTED IN}

Overview: Homogeneous nucleation from the vapor phase-The experimental science The Journal of Chemical Physics 145, 211702 (2016); https://doi.org/10.1063/1.4962283

Crystal nucleation as the ordering of multiple order parameters

The Journal of Chemical Physics 145, 211801 (2016); https://doi.org/10.1063/1.4962166

Overview: Experimental studies of crystal nucleation: Metals and colloids

The Journal of Chemical Physics 145, 211703 (2016); https://doi.org/10.1063/1.4963684

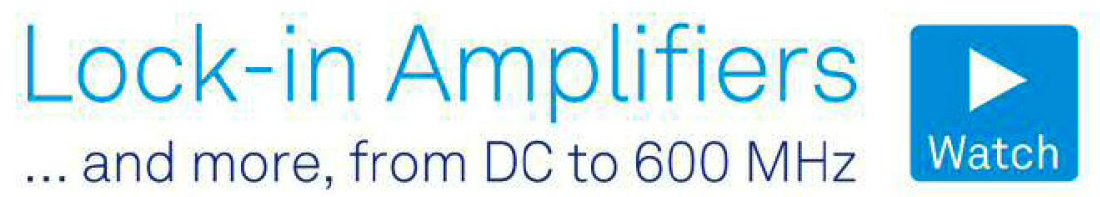




\title{
Observation of propane cluster size distributions during nucleation and growth in a Laval expansion
}

\author{
Jorge J. Ferreiro, Satrajit Chakrabarty, Bernhard Schläppi, and Ruth Signorell \\ Laboratory of Physical C'hemistry, EIH Zürich, Vladimir-Prelog Weg 2, CH-8093 Zürich, Switzerland
}

(Received 6 June 2016; accepted 18 July 2016; published online 3 August 2016)

\begin{abstract}
We report on molecular-level studies of the condensation of propane gas and propane/ethane gas mixtures in the uniform (constant pressure and temperature) postnozzle flow of Laval expansions using soft single-photon ionization by vacuum ultraviolet light and mass spectrometric detection. The whole process, from the nucleation to the growth to molecular aggregates of sizes of several nanometers $(\sim 5 \mathrm{~nm})$, can be monitored at the molecular level with high time-resolution $(\sim 3 \mu \mathrm{s})$ for a broad range of pressures and temperatures. For each time, pressure, and temperature, a whole mass spectrum is recorded, which allows one to determine the critical cluster size range for nucleation as well as the kinetics and mechanisms of cluster-size specific growth. The detailed information about the size, composition, and population of individual molecular clusters upon condensation provides unique experimental data for comparison with future molecular-level simulations. Published by AIP Publishing. [http://dx.doi.org/10.1063/1.4960050]
\end{abstract}

\section{INTRODUCTION}

The picture behind gas phase nucleation is that increasing saturation of a gas eventually leads to the formation of a critical cluster consisting of $n_{c}$ molecules. Once formed, the critical clusters spontaneously grow to larger molecular aggregates and finally to aerosol particles. The formation of the critical cluster is the rate determining step and the maximum in the Gibbs free energy occurs at $n_{\mathrm{c}}$. A true molecular-level undcrstanding of gas phasc nuclcation and subscquent growth processes, however, is still missing. Even for homogeneous nucleation in simple single component systems, experimental and theoretical nucleation rates are found to deviate by many orders of magnitude (see Refs. 1-9 and references therein). The origin of the deviations is largely unclear.

The various experimental techniques used for nucleation studies (e.g., nucleation chambers or supersonic expansions) allow the investigation of nucleation rates over $\sim 20$ orders of magnitude (see Ref. 6 and references therein). The direct measurement quantity is typically the aerosol particle number density (after nucleation and growth) as a function of the saturation $S$. The nucleation rate is then determined from the particle number density, and the critical cluster size $n_{\mathrm{c}}$ is derived from theoretical models, often from classical nucleation theory $(\mathrm{CNT})$ or variants thereof. ${ }^{7}$ In this contribution, we present measurements on propane nucleation and growth with a new apparatus, which instead of monitoring particle number densities allows us to directly record the cluster size distribution and the chemical composition of the clusters during nucleation and growth. ${ }^{10,11}$ As will be demonstrated in Section III, we thus can directly measure the critical cluster size range and follow the time evolution of individual cluster sizes during growth from small molecular aggregates to nanometer-sized particles. The detailed molecular-level information (cluster size and composition) we obtain provides information complementary to previous nucleation and growth studies (see Refs. 1-7 and references therein). In combination with future molecularlevel simulations, detailed information at the cluster level could provide deeper insight into the molecular mechanisms of nucleation and growth.

Our experimental setup combines a Laval expansion for cluster formation with soft ionization mass spectrometric detection of the cluster size distribution (Section II). In contrast to the pioneering work of Wyslouzil and co-workers, $2,5,6,12$ who studied nucleation and growth inside Laval nozzles with small angle X-ray scattering and infrared spectroscopy, we perform our investigations in the uniform postnozzle flow of the Laval nozzle using mass spectrometric detection. In the past, various research groups have exploited the postnozzle flow for the investigations of gas phase kinetics using a broad range of different characterization methods. ${ }^{13-27}$ As in these gas kinetics investigations, our cluster studies rely on the constant and well-defined temperalure $\left(T_{\mathrm{F}}\right)$ and pressure of the condensable (here $p_{\text {propane }}$ ) in the postnozzle flow. This results in a constant and well-defined saturation,

$$
S=\frac{p_{\text {propane }}}{p_{\mathrm{eq}\left(T_{\mathrm{F}}\right)}}
$$

where $p_{\mathrm{eq}}$ is the equilibrium vapour pressure of the propane gas at $T_{\mathrm{F}}$. This is a prerequisite for controlled nucleation and growth studies. In principle, nucleation can occur when $S>1$. However, a particular type of experiment, such as our supersonic expansion, defines a window of observable nucleation time scales, which means that nucleation can only be observed if it takes place within this time window. 'Throughout this paper, we use the term "subcritical" for conditions for which $S>1$ but nucleation is not observed, whereas "supercritical" refers to conditions for which nucleation has already taken place in our supersonic expansions. Note that there exists no relation to the thermodynamic notion of supercritical. 
In addition to the uniform postnozzle flow, other crucial aspects of our experimental setup concern the mass spectrometric detection. Especially after extensive growth, the cluster sizes span a wide range from small oligomers to nanometer-sized clusters (many hundred thousand mass units). To properly record the whole size distribution in one mass spectrum, we apply high voltages (up to $30 \mathrm{kV}$ ) to the ion optics..$^{10,11,28}$ The high voltages are also crucial for the sensitive detection of the nucleation event since the number densities of clusters are very low at the point where nucleation sets in. Another major issue is the soft ionization of the weakly bound molecular aggregates. Substantial cluster fragmentation upon ionization would completely falsify nucleation and growth results. For this reason, we employ single-photon ionization with a home-built, low photon flux vacuum ultraviolet (VUV) laser at energies just above the lowest ionization threshold. ${ }^{10,11,29,30}$ By comparison with the soft "Na-doping method," $28.31-33$ wc have shown in previous studies that single-photon VUV ionization is a soft ionization method for substances, such as propane, that are not prone to extensive intracluster chemistry. ${ }^{34}$ Similar results regarding soft VUV ionization of clusters were reported by other groups ${ }^{35,36}$ It is important to note that electron ionization in particular is unsuitable for nucleation and growth studies because it causes substantial cluster fragmentation as shown by Buck and Fárnik and co-workers. ${ }^{31,32,37}$ This would severely falsify the results. For the example of a water cluster with mean size of 138 molecules, Fárník and co-workers reported fragmentation upon electron ionization to a mean cluster size of 13 molecules, i.e., a measured cluster size that is only $10 \%$ of the true cluster size. With single-photon VUV ionization, by contrast, the original cluster size stays largely intact as demonstrated in Refs. 34 and 35.

The present paper summarizes the first systematic nucleation and growth studies with our new Laval setup. In our previous papers, we have reported on the details of the setup and its performance, and we have presented first nucleation and growth results. ${ }^{10,11}$ The experimental setup used in this study has been improved in comparison with the version used in the previous studies, in particular with respect to the precision of the mechanical parts (nozzle translation stage). As discussed in Ref. 11, the interpretation of the detailed experimental cluster data is not straightforward, especially for nucleation, and will require comparison with molecular-level simulations.

\section{EXPERIMENT}

Fig. 1 shows a simplified scheme of our pulsed Laval setup, which has been described in detail in Refs. 10 and 11 . Here, we provide only a short summary. Neutral, weakly bound propane (ethane) aggregates are formed in pulsed Laval expansions and are detected by soft ionization mass spectrometry. The stagnation volume of the Laval nozzle (stagnation temperature $T_{0}$, stagnation pressure $p_{0}$ ) is fed by two solenoid valves (not shown) with nominal pulse durations of $\sim 6 \mathrm{~ms}$. The solenoid valves are connected to a gas reservoir (not shown) containing the sample gas mixture that consists of a condensable gas and a carrier gas. The gas supply to

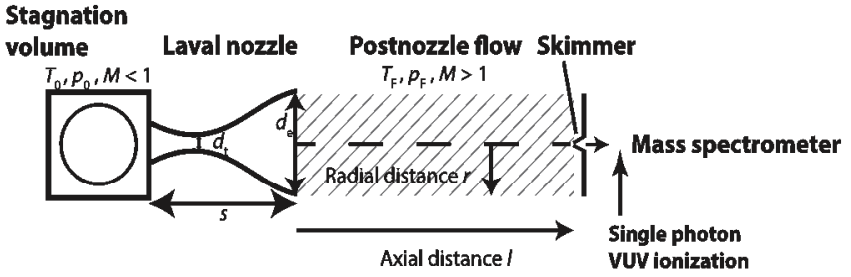

FIG. 1. Sketch of the experimental setup, which consists of a Laval nozzle for cluster formation and soft mass spectrometric detection. $T_{0}$ and $p_{0}$ are the stagnation temperature and pressure, respectively. $M$ is the Mach number. $s$, $d_{\mathrm{t}}$, and $d_{\mathrm{e}}$ are the lengths of the Laval nozzle, the throat diameter, and the exit diameter, respectively. $T_{\mathrm{F}}$ and $p_{\mathrm{F}}$ are the flow temperature and pressure, respectively. $l$ and $r$ are the axial and radial distances, respectively. $l$ is the distance between nozzle exit and skimmer. Soft single-photon ionization of the clusters is performed with photons from a home-built VUV laser. The cluster distributions are detected by a linear time- of-flight mass spectrometer.

the reservoir is regulated by mass flow controllers. For the experiments reported here, we used Ar (carrier gas; PanGas, 5.0), $\mathrm{N}_{2}$ (carrier gas; PanGas, 5.0), $\mathrm{C}_{3} \mathrm{H}_{8}$ (condensable gas; Messer, 3.5), and $\mathrm{C}_{2} \mathrm{H}_{6}$ (second condensable gas in binary mixture; Messer, 3.5).

The convergent-divergent shape of the Laval nozzle produces a flow with constant Mach number $M$ (constant flow temperature $T_{\mathrm{F}}$ and constant flow pressure $p_{\mathrm{F}}$ ) at the nozzle exit. The uniform flow is maintained in the postnozzle region over $\sim 10$ to $20 \mathrm{~cm}$ by matching the background pressure $p_{\text {exp }}$ in the expansion chamber to $p_{\mathrm{F}}$ (static pressure felt in the flow frame). The flow uniformity is characterized by impact pressure $\left(p_{\mathrm{I}}\right)$ measurements in the postnozzle flow region as a function of the axial $(l)$ and radial $(r)$ distances. ${ }^{10,13-15,25,38,39}$ The pressure $p_{0}$ in the stagnation volume and impact pressure $p_{\mathrm{I}}$ in the postnozzle flow are measured by pressure transducers. The Mach number $M$ is determined from the ratio of $p_{\mathrm{I}}$ and $p_{0}$ and the heat capacity ratio $\gamma$ of the sample gas mixture using the Rayleigh-Pitot formula. ${ }^{13}$ Once $M$ is known, the flow temperature $T_{\mathrm{F}}$, the flow pressure $p_{\mathrm{F}}$, and the number density $n_{\mathrm{F}}$ of the gas in the postnozzle flow are determined assuming isentropic flow of an ideal gas (see formulas in Refs. 10 and 13). The dimensions of the different Laval nozzles used in this study and the characteristics of the corresponding Laval expansions are summarized in Table I. The axially averaged Mach number $\bar{M}$ is the average of the Mach number along the flow axis from $l=0$ (nozzle exil) $t o l=l_{\max }$, typically averaged over 10-15 diferent axial distances $l . \bar{M}$ and its standard deviation (1o) yield the axially averaged flow temperature $\bar{T}_{F}$ and the corresponding standard deviation, which are used as an indicator for the quality of the postnozzle flow. ${ }^{10}$ Table I shows that the Mach 4.0 nozzle yields the most uniform postnozzle flow, followed by the Mach 4.5 nozzle. The poorest flow quality is found for the Mach 3.5 nozzle. $\bar{T}_{\mathrm{F}}$ is the important temperature for the growth measurements reported in Section III C, where the growth is observed as a function of $l$ along the flow axis. For the nucleation experiments in Sections III A and III B, $\bar{T}_{\mathrm{F}}$ and its standard deviation are not the relevant quantities because these experiments are performed at a fixed axial distance of $l=60 \mathrm{mmm}$. Therefore, we determine a positionally averaged flow temperatures $T_{\mathrm{F}}$ with standard deviation, which corresponds to the average and one standard deviation for 40 
TABLE I. Dimensions of the Laval nozzles (see also Fig. 1): $s$ is the length of the Laval nozzle, $d_{\mathrm{t}}$ is the throat diameter of the nozzle, and $d_{\mathrm{e}}$ is the exit diameter of the nozzle. A mixture of Ar carrier gas and propane gas was used at a constant stagnation temperature of $T_{0}=293 \mathrm{~K}$. Characteristics of the postnozzle flow: $p_{\mathrm{F}}$ is the flow pressure, $p_{\text {propane }}$ is the propane partial pressure, $l_{\max }$ is the length of the uniform postnozzle flow, $\bar{M}$ is the axially averaged Mach number with standard deviation, $\bar{T}_{\mathrm{F}}$ is the axially averaged flow temperature with standard deviation, and $T_{\mathrm{F}}$ is the positionally averaged flow temperature with standard deviation. The data correspond to the respective data for $p_{\mathrm{N}_{2}}=0$ Pa in Table II.

\begin{tabular}{lccc}
\hline \hline & Mach 4.5 & Mach 4.0 & Mach 3.5 \\
\hline$s / \mathrm{mm}$ & 79.5 & 84.0 & 47.4 \\
$d_{\mathrm{t}} / \mathrm{mm}$ & 6.2 & 6.6 & 8.0 \\
$d_{\mathrm{e}} / \mathrm{mm}$ & 22.6 & 21.7 & 22.2 \\
$p_{\mathrm{F}} / \mathrm{Pa}$ & 35 & 40 & 27 \\
$p_{\mathrm{propane}} / \mathrm{Pa}$ & 0.175 & 0.280 & 0.540 \\
$l_{\mathrm{max}} / \mathrm{mm}$ & 150 & 150 & 100 \\
$\bar{M}$ & $4.32 \pm 0.14$ & $3.98 \pm 0.06$ & $3.52 \pm 0.20$ \\
$\bar{T}_{\mathrm{F}} / \mathrm{K}$ & $41.3 \pm 2.0$ & $47.5 \pm 1.2$ & $54.6 \pm 4.6$ \\
$T_{\mathrm{F}} / \mathrm{K}$ & $42.1 \pm 0.4$ & $49.7 \pm 0.7$ & $55.4 \pm 1.0$ \\
\hline \hline
\end{tabular}

different measurements recorded at $l=60 \mathrm{~mm}$. Table I reveals that $T_{\mathrm{F}}$ has a substantially lower standard deviation than $\bar{T}_{\mathrm{F}}$, which proved important for the nucleation experiments, where $T_{\mathrm{F}}$ is varied in steps of less than $1 \mathrm{~K}$.

The cluster size distribution in the postnozzle flow is detected by soft ionization mass spectrometry. For this purpose, the central part of the postnozzle flow is sampled by a skimmer ( $1 \mathrm{~mm}$ ) before entering the time-of-flight (TOF) mass spectrometer. The molecular aggregates are then ionized by single-photons close to the lowest ionization threshold of the clusters. For the current study, we use VUV photons of $13.3 \mathrm{eV}$ ( $92.8 \mathrm{~nm})$. The VUV light is provided by a home-built, tunable, low-flux $\left(10^{10}\right.$ photons per pulse at $\left.20 \mathrm{~Hz}\right)$, nanosecond VUV laser. ${ }^{10,11,29,30}$ As emphasized in the introduction, singlephoton VUV ionization is absolutely crucial for nucleation and growth studies to ensure soft ionization of the weakly bound molecular aggregates. We again stress that electron ionization is particularly unsuitable for such studies because it destroys the original cluster distribution (see comparisons of different ionization techniques in Refs. 31-34 and 37). The ionized aggregates are accelerated by a Wiley-McLaren type time-offlight (TOF) configuration and detected by a microchannel plate (MCP) detector. An imporlant fealure of our home-built mass spectrometer is the possibility of recording cluster ion signals ranging from the dimer to aggregates with hundreds of thousands of mass units in the same mass spectrum. This is particularly important for the growth studies. The difficulty of detecting heavy aggregates with MCP detectors is overcome by applying extraction voltages of up to $30 \mathrm{kV}$ to the ion optics. ${ }^{10,28}$ We typically work at conditions, for which the number of gas phase propane monomers by far outnumbers the number of propane molecules in clusters (see Section III A 2). At MCP voltage settings optimized for cluster detection (high voltages), the monomer signal would thus cause saturation effects. To avoid these, the monomer is selectively deflected by a pulsed electrical deflection plate that is located $\sim 20 \mathrm{~cm}$ in front of the MCP detector.

\section{RESULTS AND DISCUSSION}

\section{A. Nucleation of pure propane}

\section{Cluster signals}

Nucleation rates and the onset of nucleation sensitively depend on the saturation $S$ (Refs. 5, 7, 11, and 40 and references therein) and therefore, according to Eq. (1), on the partial pressure of the condensable gas (here $p_{\text {propane }}$ ) and, through the vapor pressure $p_{\text {vap }}$ of the condensable gas, on the flow temperature $T_{\mathrm{F}}$ in the postnozzle flow. We have demonstrated in Ref. 11 that the onset of nucleation and the critical cluster size range can be extracted directly from the experimental evolution of the cluster size distribution as a function of $S$. When $S$ is gradually increased, a step-like increase in the average and the largest cluster size is observed as soon as nucleation sets in (see Figs. 4-6 in Ref. 11). In Ref. 11, we have mainly reported on nucleation studies where $S$ was changed systematically by increasing $p_{\text {propane }}$ at constant $T_{\mathrm{F}}$. The onset of nucleation was observed for a change in $p_{\text {propane }}$ of only $0.1 \%$, which demonstrates that $p_{\text {propane }}$ and $T_{\mathrm{F}}$ must be well-defined and well-controlled. Here, we show that an equivalent step-like increase in the average and the largest cluster size is observed upon nucleation when $T_{\mathrm{F}}$ decreases at constant $p_{\text {propane. }}$ The variation of $T_{\mathrm{F}}$ in steps of less than $1 \mathrm{~K}$ is achieved by replacing a small percentage of the Ar carrier gas by $\mathrm{N}_{2}$ carrier gas (see typical values of $p_{F}$ and $p_{\mathrm{N}_{2}}$ in Table II).

Fig. 2 shows a typical nucleation experiment for the Mach 4.0 nozzle (Tables I and II), for which $T_{\mathrm{F}}$ is gradually increased by increasing $p_{\mathrm{N}_{2}}$ at a constanl propane pressure of $p_{\text {propane }}=0.28 \mathrm{~Pa}$ and a constant flow pressure of $p_{\mathrm{F}}=40 \mathrm{~Pa}$. Panels (a) and (b) show how the largest cluster size $n_{\max }$ evolves with increasing saturation $S . n_{\max }$ is the number of molecules in the largest cluster observed in each cluster distribution, shown as a function of the $\mathrm{N}_{2}$ concentration (panel (a)) and the flow temperature $T_{\mathrm{F}}$ (panel (b)). Note that the $\mathrm{N}_{2}$ concentration and $T_{\mathrm{F}}$ are two independent measurement quantities in our experiment. The $\mathrm{N}_{2}$ concentration is determined from $p_{\mathrm{N}_{2}}$ and $p_{\mathrm{F}}$, while $T_{\mathrm{F}}$ is determined from impact pressure measurements (Section II). Figs. 2(a) and 2(b) show a step-like increase in $n_{\max }$, which we interpret as the onset of nucleation in accordance with our previous study in Ref. 11. Note that $n_{\max }$ is used here to represent the onset of nucleation, but that the same qualitative trends are observed for the average cluster size or the central value (defined below). The small changes in the $\mathrm{N}_{2}$ concentration $(\sim 0.2 \%)$ and in $T_{\mathrm{F}}$ $(\sim 0.4 \mathrm{~K})$ across the onset show the sensitivity of nucleation to experimental conditions, which demonstrates the importance of well-defined and stable conditions. Table II also reports equivalent data for the other two Mach nozzles (4.5 and 3.5). The data show that the critical flow temperature $I_{\mathrm{F}, \mathrm{c}}$ can be determined within approximately 1-2 standard deviations of $T_{\mathrm{F}}$. For the Mach 4.0 nozzle this amounts to $\sim \pm 0.6 \mathrm{~K}$.

The onset of nucleation as a function of the $\mathrm{N}_{2}$ concentration is very sharp (Fig. 2(a)), while the onset as a function of $T_{\mathrm{F}}$ is smeared out (panel (b)). The reason is the higher precision of the $\mathrm{N}_{2}$ concentration measurements compared with the flow temperature measurements. For 
TABLE II. Experimental results for nucleation in three different Laval nozzles. $p_{\mathrm{F}}$ and $T_{\mathrm{F}}$ are the flow pressure and temperature, respectively, $p_{\text {propane }}$ is the propane partial pressure, and $p_{\mathrm{N}_{2}}$ and $\% \mathrm{~N}_{2}$ are the nitrogen partial pressure and the nitrogen concentration, respectively. $p_{\mathrm{N}_{2}}$ has a typical standard deviation (lo) of $\sim 0.05 \mathrm{~Pa} . n_{\max }$ is the largest cluster size detected in each mass spectrum, $n_{\mathrm{M}}=\frac{n_{\min }+n_{\max }}{2}$ is the central value of the cluster size in each mass spectrum, $\left[n_{\max }, n_{\mathrm{M}}\right]$ is the critical cluster size range (see text), $\bar{n}_{\mathrm{c}}$ is the average critical cluster size, and $n_{\mathrm{c}}^{\mathrm{CNT}}$ is the critical cluster size calculated from CNT.

\begin{tabular}{|c|c|c|c|c|c|c|c|}
\hline Nozzle & $p_{\mathrm{N}_{2}} / \mathrm{Pa}\left(\% \mathrm{~N}_{2}\right)$ & $\left(T_{\mathrm{F}} \pm \sigma\right) / \mathrm{K}$ & $n_{\max }$ & $n_{\mathrm{M}}$ & {$\left[n_{\max }, n_{\mathrm{M}}\right]$} & $\bar{n}_{\mathrm{c}}$ & $\boldsymbol{n}_{\mathrm{c}}^{\mathrm{CNT}}$ \\
\hline \multicolumn{8}{|c|}{ Mach 4.5} \\
\hline \multirow{2}{*}{\multicolumn{8}{|c|}{$\begin{array}{l}p_{\text {propane }}=0.175 \mathrm{~Pa} \\
p_{\mathrm{F}}-35 \mathrm{~Pa}\end{array}$}} \\
\hline & & & & & & & \\
\hline & $0.00(0.00 \%)$ & $42.1 \pm 0.4$ & 51 & 26 & \multirow{11}{*}[2,18]{} & \multirow{11}{*}{10} & \multirow{11}{*}{2} \\
\hline & $0.35(1.00 \%)$ & $42.8 \pm 0.4$ & 34 & 18 & & & \\
\hline & $0.70(2.00 \%)$ & $43.1 \pm 0.4$ & 31 & 16 & & & \\
\hline & $1.05(3.00 \%)$ & $42.9 \pm 0.4$ & 37 & 19 & & & \\
\hline & $1.40(4.00 \%)$ & $43.6 \pm 0.4$ & 34 & 18 & & & \\
\hline & $1.15(5.00 \%)$ & $44.1 \pm 0.4$ & 35 & 18 & & & \\
\hline & $1.79(5.11 \%)$ & $43.6 \pm 0.4$ & 2 & 2 & & & \\
\hline & $1.82(5.20 \%)$ & $43.8 \pm 0.4$ & 2 & 2 & & & \\
\hline & $1.93(5.51 \%)$ & $43.6 \pm 0.4$ & 1 & 1 & & & \\
\hline & $2.10(6.00 \%)$ & $43.9 \pm 0.4$ & 1 & 1 & & & \\
\hline & $3.50(10.00 \%)$ & $44.3 \pm 0.4$ & 1 & 1 & & & \\
\hline \multicolumn{8}{|c|}{ Mach 4.0} \\
\hline \multicolumn{8}{|c|}{$\begin{array}{l}p_{\text {propane }}=0.280 \mathrm{~Pa} \\
p_{\mathrm{F}}=40 \mathrm{~Pa}\end{array}$} \\
\hline & $0.00(0.00 \%)$ & $49.7 \pm 0.7$ & 79 & 40 & \multirow{10}{*}[3,24]{} & \multirow{10}{*}{14} & \multirow{10}{*}{3} \\
\hline & $0.80(2.00 \%)$ & $49.8 \pm 0.7$ & 70 & 36 & & & \\
\hline & $1.20(3.00 \%)$ & $50.3 \pm 0.5$ & 56 & 29 & & & \\
\hline & $2.00(5.00 \%)$ & $50.5 \pm 0.7$ & 50 & 26 & & & \\
\hline & $2.40(6.00 \%)$ & $50.8 \pm 0.5$ & 46 & 24 & & & \\
\hline & $2.80(7.00 \%)$ & $51.1 \pm 0.6$ & 47 & 24 & & & \\
\hline & $2.88(7.20 \%)$ & $51.5 \pm 0.5$ & 3 & 2 & & & \\
\hline & $3.00(7.50 \%)$ & $51.4 \pm 0.5$ & 3 & 2 & & & \\
\hline & $3.20(8.00 \%)$ & $51.1 \pm 0.6$ & 1 & 1 & & & \\
\hline & $4.00(10.00 \%)$ & $51.8 \pm 0.7$ & 1 & 1 & & & \\
\hline \multicolumn{8}{|c|}{ Mach 3.5} \\
\hline \multicolumn{8}{|c|}{$\begin{array}{l}p_{\text {propane }}=0.540 \mathrm{~Pa} \\
p_{\mathrm{F}}=27 \mathrm{~Pa}\end{array}$} \\
\hline & $0.00(0.00 \%)$ & $55.4 \pm 1.0$ & 56 & 29 & & \multirow{9}{*}{8} & \multirow{9}{*}{3} \\
\hline & $0.21(0.78 \%)$ & $55.2 \pm 1.1$ & 43 & 22 & \multirow{8}{*}[3,12]{} & & \\
\hline & $0.27(1.00 \%)$ & $55.2 \pm 0.9$ & 38 & 20 & & & \\
\hline & $0.41(1.52 \%)$ & $55.3 \pm 1.1$ & 33 & 17 & & & \\
\hline & $0.54(2.00 \%)$ & $55.6 \pm 1.0$ & 28 & 15 & & & \\
\hline & $0.59(2.19 \%)$ & $55.8 \pm 1.0$ & 23 & 12 & & & \\
\hline & $0.62(2.30 \%)$ & $55.2 \pm 0.9$ & 3 & 2 & & & \\
\hline & $0.70(2.60 \%)$ & $55.5 \pm 1.0$ & 3 & 2 & & & \\
\hline & $1.35(5.00 \%)$ & $56.4 \pm 1.1$ & 1 & 1 & & & \\
\hline
\end{tabular}

completeness, Fig. 2(c) shows the correlation between the $T_{\mathrm{F}}$ and the $\mathrm{N}_{2}$ concentration for the experimental values (open circles) and for a simulation (closed triangles) for the Mach 4.0 nozzle. The computational fluid dynamics (CFD) simulation was performed with ANSYS Fluent 14.5 with the nozzle dimensions from Table I and the experimental partial pressures (Table II) as input parameters. The experimental data show an almost linear decrease of $T_{\mathrm{F}}$ with increasing $\mathrm{N}_{2}$ concentration, while the simulation predicts a perfect linear dependence. The absolute $T_{\mathrm{F}}$ values in the simulation are slightly lower than the experiment. Such differences in the absolute value seem to be a common phenomenon for Laval expansions. ${ }^{10}$ The fact that the experiment and the simulation show linear trends and the fact that the onset of nucleation as a function of the $\mathrm{N}_{2}$ concentration is much sharper than the onset as a function of $T_{\mathrm{F}}$ as derived from impact pressure measurements imply that the true $T_{\mathrm{F}}$ is actually much more stable. According to the simulated curve, an uncertainty in the $\mathrm{N}_{2}$ concentration of $0.13 \%(\sim 0.05 \mathrm{~Pa})$ would correspond to a change in $T_{\mathrm{F}}$ of only $0.04 \mathrm{~K}$, which lies far below the experimental standard deviation of $\pm 0.6 \mathrm{~K}$. This suggests that calibration curves similar to the one in Fig. 2(c) would allow for a much more precise determination of the true $T_{\mathrm{F}, \mathrm{c}}$ in future investigations.

The measured mass spectra as a function of $S$ allow us to retrieve a range for the critical cluster size $n_{\mathrm{c}}$. For this 

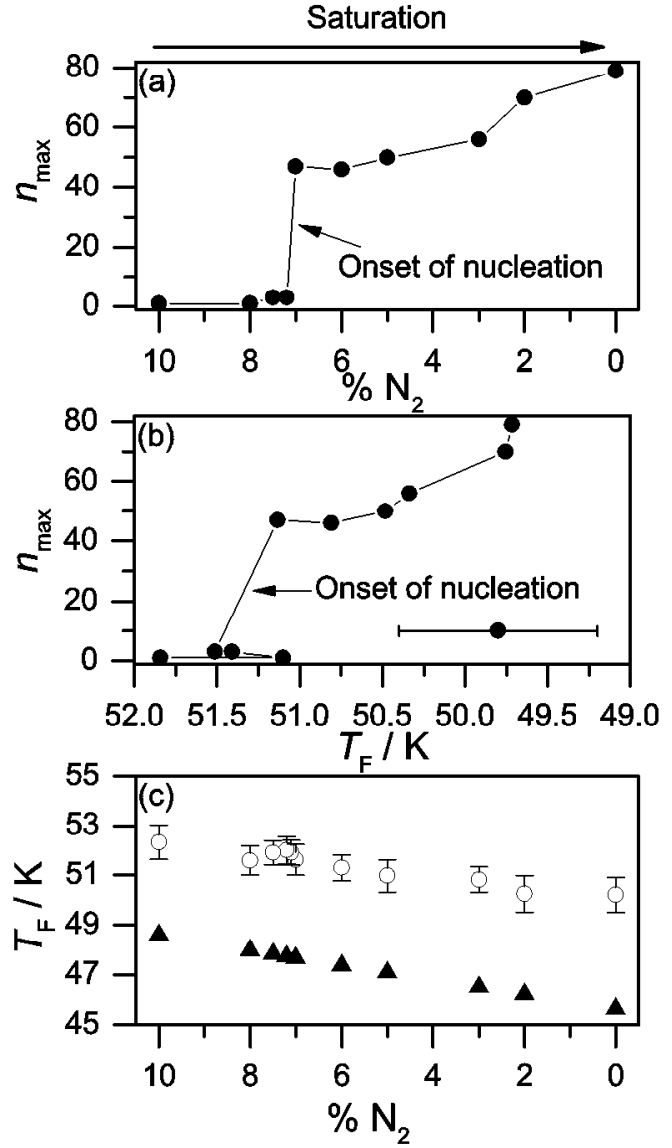

FIG. 2. Nucleation experiment for the Mach 4.0 nozzle summarized in Table II. (a) The largest cluster size $n_{\max }$ that is detected in the mass spectra as a function of the nitrogen concentration. (b) The same as for panel (a), but as a function of the flow temperature $T_{\mathrm{F}}$. The error bar indicates a typical standard deviation in $T_{\mathrm{F}}$. (c) $T_{\mathrm{F}}$ as a function of the concentration. Open circles: Experimental data with standard deviation. Closed triangles: $T_{\mathrm{F}}$ values calculated with ANSYS Fluent.

purpose, we follow a similar approach as proposed in Ref. 11. First, we determine $n_{\max }$ for the last mass spectrum measured under subcritical conditions just before nucleation sets in. This number provides a lower limit for $n_{\mathrm{c}}$. For the Mach 4.0 example in Fig. 2 and Table II, this corresponds to the mass spectrum recorded at $7.2 \% \mathrm{~N}_{2}$ with $n_{\max }=3$. As an upper limit, we propose to use the central value $n_{\mathrm{M}}=\frac{n_{\min }+n_{\max }}{2}$ deternined for the first mass spectrum measured under supercritical conditions just after nucleation has set in, where $n_{\min }$ is the smallest cluster size that is observed in this first supercritical mass spectrum. $n_{\mathrm{M}}$ is not a rigorous upper limit because as soon as nucleation occurs, cluster growth happens spontaneously (Section III C), so that the $n_{\mathrm{M}}$ presumably somewhat overestimates the true upper limit. For the Mach 4.0 example, we determine $n_{\mathrm{M}}=24$. This results in a critical cluster size range of $\left[n_{\max }, n_{\mathrm{M}}\right]=[3,24]$ with an average value of $\bar{n}_{\mathrm{c}}=14$.

Table II lists the values for $n_{\max }, n_{\mathrm{M}},\left[n_{\max }, n_{\mathrm{M}}\right]$, and $\bar{n}_{\mathrm{c}}$ for the experiments with the three different nozzles. The critical cluster size ranges $\left[n_{\max }, n_{\mathrm{M}}\right]$ are very similar for the three different nozzles. This might be a consequence of the very high values for the critical saturations $S_{\mathrm{c}}$ (between $\sim 10^{12}$ and $\sim 10^{18}$ using the data in Ref. 41 for extrapolation). Such high saturations immediately raise the question of whether nucleation occurs in the presence of a free energy barrier or whether the process is barrierless (spinodal decomposition). ${ }^{7}$ Typically, the critical cluster size $n_{\mathrm{c}}$ is described as a single cluster size. Depending on the conditions it ranges from several to tens of molecules (Refs. 3, 11, and 40 and references therein). However, as discussed by Kalikmanov ${ }^{7}$ the notion of a single critical nucleus does not yield a complete picture. One should rather discuss a critical region $\left[n_{\mathrm{c}}, n\right]$ where the nucleation barrier varies within the average thermal energy $k_{\mathrm{B}} T$ (i.e., $\Delta G\left(n_{\mathrm{c}}\right)-\Delta G(n) \leq k_{B} \cdot T$ ). In our experiments, $k_{\mathrm{B}} T_{\mathrm{F}, \mathrm{c}}$ lie between 6.0 and $7.6 \times 10^{-22} \mathrm{~J}$ for the three nozzles. If the processes in the three different nozzles (Table II) are barrierless or all barriers are similar within $k_{\mathrm{B}} T_{\mathrm{F}}$, one would thus not expect to observe a difference in $\left[n_{\max }, n_{\mathrm{M}}\right]$ for the three different nozzles, as found in the experiment. Note that classical nucleation theory (CNT) cannot provide any further insight here since in the framcwork of CNT the barrier never vanishes, which is unphysical (see below). To better understand the process, we are currently examining kinetic approaches to nucleation and growth in the deeply supercooled and spinodal regions, including the use of Dynamical Nucleation Theory (DNT) ${ }^{42,43}$ and using molecular dynamics simulations to follow the time evolution of the cluster size distributions. These results will be compared to the predictions of extensions of CNT applicable to the spinodal region. ${ }^{44,45}$

For completeness, we provide in the following some estimates using CNT. The critical cluster size $n_{\mathrm{c}}^{\mathrm{CNT}}$ can be calculated from the reduced surface tension $\theta_{\infty}$ and the critical saturation $S_{\mathrm{c}}{ }^{7}$ as

$$
n_{\mathrm{c}}^{\mathrm{CNT}}=\left[\frac{2}{3} \frac{\theta_{\infty}\left(T_{\mathrm{F}, \mathrm{c}}\right)}{\ln S_{\mathrm{c}}}\right]^{3}
$$

where $\theta_{\infty}\left(T_{\mathrm{F}, \mathrm{c}}\right)$ is calculated from the planar surface tension $\gamma_{\infty}\left(T_{\mathrm{F}, \mathrm{c}}\right)$, the surface area of the monomer, and the thermal energy $k_{\mathrm{B}} T_{\mathrm{F}, \mathrm{c}}$. The planar surface tension decreases with temperature according to Ref. 46, which is valid in a temperature range of $253-333 \mathrm{~K}$. Here, we use the same formula for extrapolation down to $T_{\mathrm{F}, \mathrm{c}}$. For the surface area of the monomer, we also use the bulk density extrapolated to $T_{\mathrm{F}, \mathrm{c}}$ according to the formula in Ref. 41. Similarly, for $S_{\mathrm{c}}=\frac{p_{\text {propane }}}{p_{\mathrm{eq}}\left(T_{\mathrm{F}, \mathrm{c}}\right)}$, the Wagner equation is used to determine $p_{\mathrm{eq}}$ at $T_{\mathrm{F}, \mathrm{c}}{ }^{41}$ Table II lists the values of $n_{\mathrm{c}}^{\mathrm{CNT}}$ for the three different experiments. The values are all very similar because of the high saturation (see Eq. (2)). They also seem to agree well with the experimental $n_{\max }$ values. However, this apparent agreement is probably meaningless because CNT is not valid at such high saturation as the following estimate of the nucleation barrier reveals. In the framework of CNT, the nucleation barrier is calculated as

$$
\Delta G\left(n_{\mathrm{c}}^{\mathrm{CNI} I}\right)=\frac{1}{3} \gamma_{\infty}\left(T_{\mathrm{F}, \mathrm{c}}\right) A\left(n_{\mathrm{c}}^{\mathrm{CNI} I}\right)
$$

where $A\left(n_{\mathrm{e}}^{\mathrm{CNT}}\right)$ is the surface area of the critical nucleus, which is determined from the bulk density extrapolated according to Ref. 41. This results in a barrier height of $\sim 44 k_{\mathrm{B}} T_{\mathrm{F}, \mathrm{c}}$ for all three nozzles, which seems unreasonably high considering the high saturations and shows the limitations of CNT. For this reason, we also abstain from determining any nucleation rates 
using CNT. Deviations between experimental results and CNT predictions at high saturation have been reported for various systems. $^{2-6}$

\section{Monomer signal}

Controlled and well-known conditions are pre-requisites for the molecular-level nucleation studies presented in this and our previous paper. ${ }^{11}$ For the present investigation, where nucleation is induced by a variation of less than $1 \mathrm{~K}$ in $T_{\mathrm{F}}$, it is important to ensure that $p_{\text {propane }}$ stays constant across this regime. This is only possible if the fraction of propane monomer that condenses into clusters upon nucleation is negligible compared with the total amount of propane monomer. Under these conditions, the monomer signal should not change upon nucleation. We show in the following that for our nucleation studies this is fulfilled to a very good approximation.

As the monomer signal is much stronger than the clusters signal (see Section II), it is not possible to record both signals with the same voltage settings for the MCP detector. To estimate the ratio $R$ of the number of gas phase monomer to the number of molecules in clusters in the postnozzle flow, we have thus performed a series of calibration studies. From these studies, we estimate $R$ to be in the range $10^{4}-10^{5}$ before nucleation. The number of monomers that condense into clusters when crossing the onset of nucleation increases typically by about a factor of 10 . The corresponding decrease in the gas phase monomer signal of $0.1 \%-0.01 \%$ is indeed negligible, so that the assumption of a constant propane partial pressure is well fulfilled in our nucleation experiments. We have also directly monitored the absolute monomer signal as a function of $T_{\mathrm{F}}$ under identical conditions as for the three nucleation experiments in Table II, but at much lower MCP voltages. Note that cluster signals cannot be detected at these low MCP voltages. As an example, Fig. 3 shows the monomer signal as a function of increasing $T_{\mathrm{F}}$ (decreasing $S$ ) recorded during nucleation experiments with the Mach 4.5 nozzle (Table II). The signal fluctuation of $\sim 17 \%(2 \sigma)$ arises solely from the intensity fluctuations of the VUV light and is unrelated to the monomer abundance. Since no monomer depletion is detected within the fluctuations, any monomer depletion must be less than $\sim 17 \%$, which is at least consistent with the estimated $0.1 \%-0.01 \%$ quoted above. Note that normalization of the monomer signal to the VUV intensity is in principle possible, but would require a VUV detector different from the one used in this study.

As will be pointed out in Section III C, monomer depletion becomes pronounced in our experiments only at the late stage of cluster growth, when multimodal size distributions are observed (typically for $n_{\max }>1000$ (Fig. 5)). At this stage of growth, the monomer depletion needs to be monitored and taken into account to come to quantitative conclusions. The observation of monomer depletion was exploited in previous cluster studies in various contexts. For example, condensation experiments performed on methanol ${ }^{12}$ as well as dimerization studies on polyaromatic hydrocarbons ${ }^{22,24}$ studied monomer depletion during cluster formation. Laksmono et al. ${ }^{12}$ observed up to $30 \%$ of the monomer undergoing cluster formation

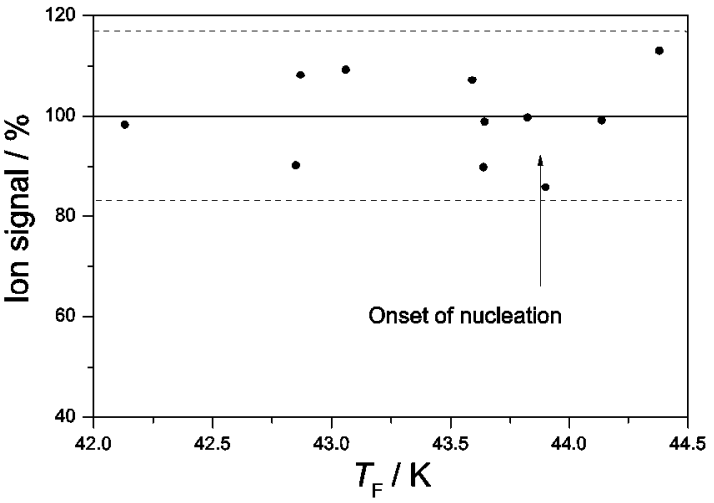

FIG. 3. Monomer signal as a function of the flow temperature $T_{\mathrm{F}}$ for the experiment with the Mach 4.5 nozzle described in Table II. The results for the other nozzles are equivalent. The dashed lines indicate $2 \sigma$.

before condensing into the liquid phase, whereas Biennier et al. ${ }^{22}$ worked in a regime where the monomer consumption remained below $15 \%$ to avoid cluster growth.

\section{B. Nucleation of propane-ethane mixtures}

Gas phase nucleation of binary systems has been investigated for, e.g., ethanol/water, ${ }^{47,48}$ ethanol/hexanol, ${ }^{49}$ and $n$-nonane/methane ${ }^{50}$ mixtures as well as in a range of theoretical studies using binary homogenous nucleation theory (BCNT) (Refs. 7 and 51-53 and references therein). Here, we very briefly report on the first binary nucleation studies of propane-ethane mixtures with our new Laval setup. We chose ethane as the second gas because the similarity of the intermolecular interactions let us expect a rather subtle effect on the nucleation behavior compared with pure propane.

Fig. 4 shows a typical binary nucleation experiment in comparison with the nucleation of pure propane and pure ethane, respectively. These experiments were performed with

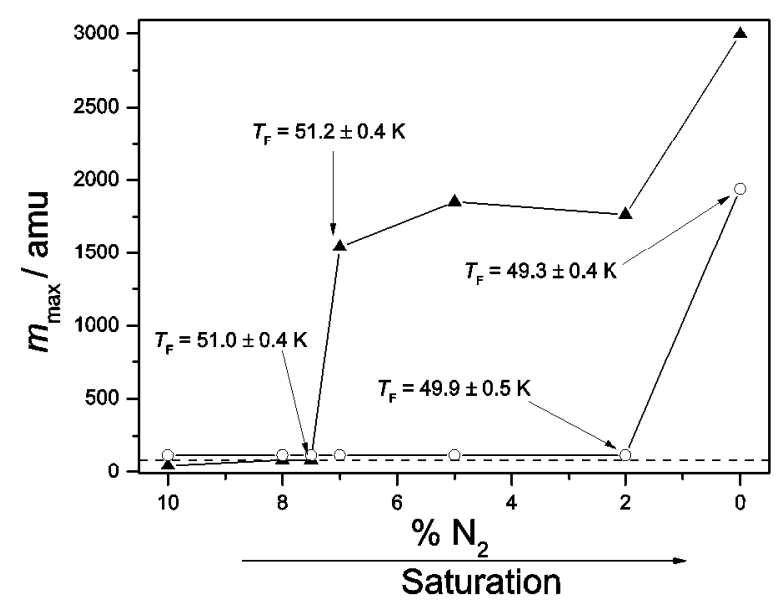

FIG. 4. Binary and unary nucleation studies in the Mach 4.0 nozzle at a flow pressure of $p_{F}=40 \mathrm{~Pa} . m_{\mathrm{mllx}}$ is the mass of the largest cluster observed in the mass spectra (assuming a single positive charge). Open circles: Binary nucleation in a propane/ethane mixture at a total partial pressure of $p_{\text {tot }}$ $=p_{\text {propane }}+p_{\text {ethane }}=0.136 \mathrm{~Pa}+0.104 \mathrm{~Pa}=0.240 \mathrm{~Pa}$. Closed triangles: Unary nucleation of propane at a propane partial pressure of $p_{\text {propane }}=0.240 \mathrm{~Pa}$. Dashed line: Experiments with pure propane at $p_{\text {propane }}=0.136 \mathrm{~Pa}$ and pure ethane at $p_{\text {ethane }} \leq 2.000 \mathrm{~Pa}$, for which nucleation is not observed. 


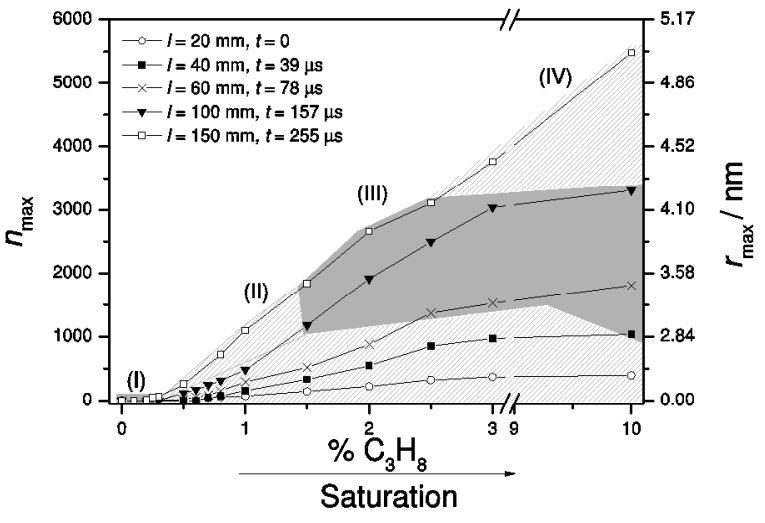

FIG. 5. Cluster growth ( $\left.n_{\max }, r_{\max }\right)$ as a function of the propane concentration $\left(\% \mathrm{C}_{3} \mathrm{H}_{8}\right)$ and the growth time $t$ (axial position $l$ ). Different growth times $t$ are indicated by different symbols. The growth is characterized by the largest cluster size $n_{\max }$ (left ordinate) or the largest cluster radius $r_{\max }$ (right ordinate) that is observed in a particular mass spectrum. $r_{\max }$ is calculated from the bulk density at $\bar{T}_{\mathrm{F}}$ assuming spherical particles. The different regions I-IV are explained in the text.

the Mach 4.0 nozzle at a flow pressure of $p_{\mathrm{F}}=40 \mathrm{~Pa}$. As for the studies in Table II, the carrier gas was an $\mathrm{Ar} / \mathrm{N}_{2}$ mixture, in which the $\mathrm{N}_{2}$ concentration was systematically varied to vary the flow temperature $T_{\mathrm{F}}$. Note that Fig. 4 shows the mass $m_{\max }$ of the largest cluster observed in each mass spectrum instead of the number of molecules $n_{\max }$ as in Fig. 2 because of the limited mass resolution (see below). The nucleation studies with pure propane (closed triangles and dashed line in Fig. 4) and pure ethane (dashed line in Fig. 4) serve as references. The closed triangles show a nucleation experiment for pure propane at a partial pressure of $p_{\text {propane }}=0.240 \mathrm{~Pa}$. The onset of nucleation is observed at $T_{\mathrm{F}, \mathrm{c}}=51.1 \mathrm{~K}$. For a propane pressure of $p_{\text {propane }}=0.136 \mathrm{~Pa}$, by contrast, nucleation is not observed over the range of nitrogen concentrations studied (dashed line at zero in Fig. 4). For pure ethane no condensation is observed for all partial pressures $p_{\text {ethane }} \leq 2.000 \mathrm{~Pa}$ (including pressures of $p_{\text {ethane }}=0.240 \mathrm{~Pa}$ and $p_{\text {ethane }}=0.104 \mathrm{~Pa}$ ). The open circles show a nucleation experiment with a propane/ethane mixture at a total partial pressure of $p_{\text {tot }}=p_{\text {propane }}+p_{\text {ethane }}=0.136 \mathrm{~Pa}+0.104 \mathrm{~Pa}$ $=0.240 \mathrm{~Pa}$. At these partial pressures, the pure substances do not condense at all. The onset of nucleation for the binary mixture is observed at $T_{\mathrm{F}, \mathrm{c}}=49.6 \mathrm{~K}$. This is $1.5 \mathrm{~K}$ lower than the onset of nucleation for pure propane at the same total pressure of $0.240 \mathrm{~Pa}$ (closed triangles), which clearly shows that the chemical composition has an influence on the nucleation behavior even for compounds with similar intermolecular interactions. As expected, a subtle change in the nucleation behavior is observed. Even though a molecular level explanation cannot be provided at this point, it seems at least reasonable for compounds with similar intermolecular interactions that the critical temperature $T_{\mathrm{F}, \mathrm{c}}$ of the mixture lies in between the $T_{\mathrm{F}, \mathrm{c}}$ of the two pure compounds at the same total partial pressure of the condensables $(0.240 \mathrm{~Pa})$. We do not observe nucleation for pure ethane at $p_{\text {ethane }}=0.240 \mathrm{~Pa}$ in the Mach 4.0 nozzle simply because at this pressure $T_{\mathrm{F}, \mathrm{c}}$ lies well below the lowest $T_{\mathrm{F}}$ attainable $(\sim 49 \mathrm{~K})$.

The limited mass resolution of our mass spectrometer $(m / \Delta m \sim 110$ at $900 \mathrm{amu} / e)$ is the reason why we cannot provide details on the composition of the clusters. For the binary system, the critical nucleus lies in the mass range of $m_{\mathrm{c}} \sim 900 \mathrm{amu}$ (singly charged clusters). The low mass resolution does not allow for an unambiguous assignment to a specific composition $\left(\mathrm{C}_{3} \mathrm{H}_{8}\right)_{n}\left(\mathrm{C}_{2} \mathrm{H}_{6}\right)_{m}$. However, many different combinations of mixed propane/ethane clusters could contribute to the mass signal around 900 amu. Because of the lack of molecular-level information and because of the deficiencies of CNT at high saturations (Section III A 1), we do not attempt to use binary classical nucleation theory (BCNT) to predict critical cluster sizes for the binary mixtures. BCNT only generalizes the concepts of CNT to even more complex systems, but does not provide any molecularlevel understanding of binary nucleation. ${ }^{7}$ A more in-depth understanding for this system requires again molecular-level simulation and more detailed experimental data providing information on cluster sizes and composition. In future studies, we plan to usc a reflectron timc-of-flight mass spectrometcr with a resolution of up to $m / \Lambda m \sim 3000$ at $900 \mathrm{amu} / e$. This should allow us to identify whether any preferential cluster compositions exist, such as, for example, a higher propane than ethane content.

\section{Cluster growth}

'I'he uniform postnozzle flow of the Laval expansion (Fig. 1) also makes it useful for cluster growth studies since the axially averaged temperature $\bar{T}_{\mathrm{F}}$ is constant over the distance $l_{\max }$ after the nozzle exit to a very good approximation (Table I). The combination with soft ionization mass spectrometry provides very detailed information on the evolution of the cluster size distribution over growth times of up to many hundred microseconds with a time resolution of a few microseconds. The temporal growth processes are monitored by systematically changing the nozzle to skimmer distance $l$ from $l=0$ to $l=l_{\text {lllax }}$. In addition, the partial pressure of the condensable ( $\left.p_{\text {propane }}\right)$ is varied. This allows us to broadly map the evolution of the cluster size distribution as a function of the growth time and the concentration of the condensable. In the following, we briefly summarize some of the most prominent general features observed during cluster growth.

Fig. 5 shows as an example a coarse map of the maximum cluster size $n_{\max }$ as a function of the propane concentration $\% \mathrm{C}_{3} \mathrm{H}_{8}$ and the growth time for the Mach 4.0 nozzle (Ar carrier gas, $p_{\mathrm{F}}=40 \mathrm{~Pa}, \bar{T}_{\mathrm{F}}=47.5 \pm 1.2 \mathrm{~K}$, flow velocity $v=510 \mathrm{~m} / \mathrm{s}$ ). The growth time $t$ is varied by changing the distance $l$. It is calculated from $l$ and the flow velocity. Here, $t=0$ corresponds to the measurements at the shortest distance $l=20 \mathrm{~mm}$. In Fig. 5, the time is coarsely mapped with changes on the order of several tens of microseconds. If required, the time-resolution can be further improved to a few microseconds. The grey shaded areas with roman numbers from I to IV indicate regions where the cluster size distributions exhibit characteristic features. In region I, nucleation has not yet taken place and only small clusters up to about the trimer are observed in the mass spectra. Region II describes the first stages of growth just after nucleation, where typically unimodal size distributions are 


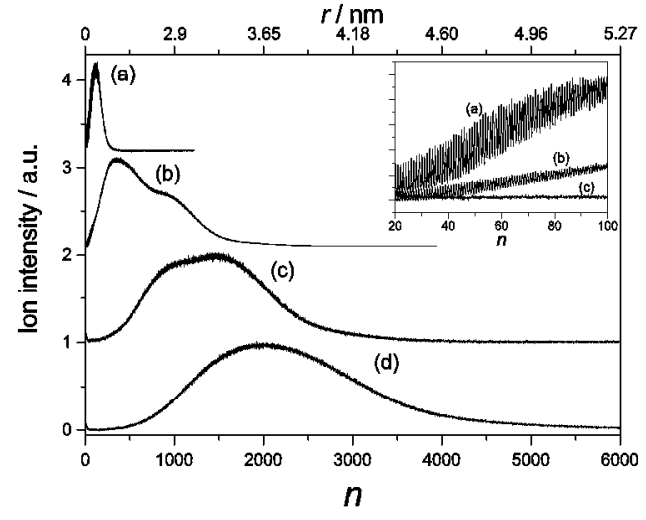

FIG. 6. Exemplary mass spectra in the different regions II-IV in Fig. 5. $n$ is the cluster size (number of molecules in a cluster). $r$ (upper abscissa) is the corresponding radius assuming bulk densities and spherical clusters. (a) Region II: for $0.8 \%$ propane $l=100 \mathrm{~mm}$. (b) Region III: for $2.5 \%$ propane $l=100 \mathrm{~mm}$. (c) Region III: for $10 \%$ propane at $l=100 \mathrm{~mm}$. (d) Region IV: for $10 \%$ propane at $l=150 \mathrm{~mm}$.

observed. An example of a unimodal distribution is provided in Fig. 6(a). The maximum cluster sizes $n_{\max }$ are usually smaller than 1000 in this region. The growth in this region seems mainly dominated by growth from the vapor phase. In region III, the cluster size distributions become multimodal with $n_{\max }$ up to $\sim 3000$. Two typical examples are depicted in Figs. 6(b) and 6(c). Together with trace (a), they demonstrate the continuous depletion of small clusters $(n<100$; see inset) with increasing average cluster size. In trace (c), the small clusters have completely disappeared. This is the most complicated growth regime in which various growth processes compete simultaneously, depleting smaller clusters and producing multimodal distributions. Larger clusters are formed by the growth of smaller clusters, the number density of which thus decreases. Simultaneously, coagulation decreases the number densities of the smaller clusters while the average cluster size increases significantly. ${ }^{54}$ Eventually this leads to region IV, where clusters become very large $n_{\max }>3000\left(r_{\max }>4 \mathrm{~nm}\right)$ and the size distributions become again unimodal (Fig. 6(d)) - a behavior that is expected from the Kelvin effect. For even longer times, when growth processes are complete and equilibrium is reached, one would expect the size distribution to become not only unimodal but also narrower due to the Kelvin effect. In our experinnent, we do not observe a pronounced narrowing, so we have probably not yet reached this limit.

Figs. 7(a)-7(c) provide a detailed view of the evolution of three different cluster sizes $n=50,300$, and 1000 as a function of time and concentration. It illustrates the cluster size specific growth behavior, which is not obvious from Figs. 5 and 6 . Small clusters $(n=50$, panel (a) ) are only present at low concentrations and early times. 'This is the regime after nucleation when the first steps of cluster growth take place. The abundance of these small clusters simply decreases as a function of time until they disappear completely. Depending on the conditions, the temporal behavior can change from a monotonous decrease with positive curvature to the one with negative curvature. The cluster $n=300$ in panel (b) behaves differently. It is present in the whole time-concentration (a)

(b)

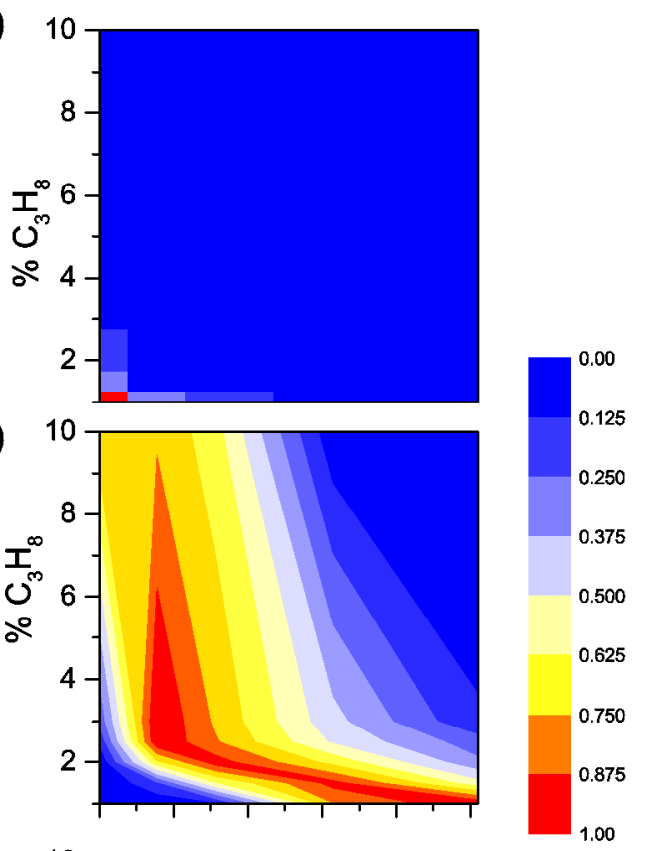

(c)

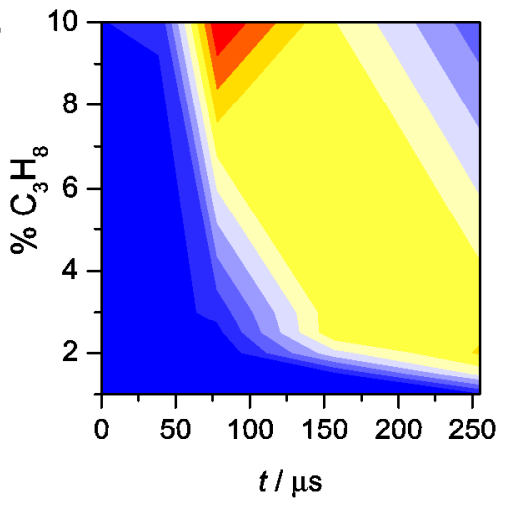

FIG. 7. Contour plots of the abundance of three different cluster sizes $n=50$, 300 , and 1000 (panels (a) (c), respectively) during cluster growth as a function of the growth time $t$ and the propane concentration $\left(\% \mathrm{C}_{3} \mathrm{H}_{8}\right)$. The three panels show size-specific cluster growth. The colour schenes correspond to the normalized cluster abundance with the abundance decreasing from red to blue.

regime. In particular in the intermediate concentration regime, the abundance of this cluster first increases with increasing time, then reaches a maximum and finally decreases again. At early times, this reflects the growth of small clusters to clusters of intermediate size $(n \sim 300)$. At later times, the abundance of these intermediate size clusters decreases again because of growth and coagulation to even larger clusters. The abundance of the largest cluster $n=1000$ (panel (c)) increases for most concentrations except at very high concentrations where the time evolution more closely resembles that of the $n=300$ cluster. Again, the functional form of the increase in abundance depends on the specific conditions. It can vary from a monotonous increase with negative curvature to one with positive curvature.

It is on purpose that we do not provide multiple lognormal fits for the cluster size distributions (Fig. 6).${ }^{54,55}$ They are not unambiguous and contain little information, so they do not provide any useful mechanistic insight. As in the case of the nucleation kinetics, detailed modelling is required to describe the growth kinetics and to unravel which mechanisms are the 
most important in a certain growth regime (I-IV in Fig. 5). Combined molecular dynamics-master equation approaches or transition state based master equation approaches hold some promise for this purpose. ${ }^{24,42}$

\section{SUMMARY}

To the best of our knowledge, the present contribution together with our previous publications ${ }^{10,11}$ report the first nucleation and cluster growth data recorded in the uniform postnozzle flow of a Laval nozzle using mass spectrometric detection after soft single-photon VUV ionization. The uniform conditions in the postnozzle flow are important to perform the nucleation and growth studies under controlled conditions. The mass spectrometric detection allows one to extract detailed molecular-level information on the size and the chemical composition of the clusters. As explained in the Introduction, the soft ionization with VUV light is crucial here. Destructive ionization methods, such as electron ionization, would distort the true cluster size distribution to such an extent that no useful nucleation and growth data could be extracted from the mass spectra. ${ }^{31,32,37}$ In contrast to previous investigations, our experiments provide cluster-size resolved information during nucleation and growth, i.e., they provide complementary molecular-level information. A direct comparison with the nucleation and growth studies in Laval nozzles performed by Wyslouzil and co-workers ${ }^{2,5,6,12}$ would be particularly interesting since they cover a similar saturation regime.

We interpret the sudden step-wise increase in the maximal cluster size that is observed with increasing saturation as the onset of nucleation and retrieve the critical saturation, critical temperature, critical concentration, and a critical cluster size range from the expansion conditions and the mass spectra at the onset of nucleation. For propane, we observe very high saturations and similar critical cluster sizes at different conditions. At these high saturations, it is not clear at all whether nucleation in the presence of a free energy barrier is observed or whether the process is barrierless (spinodal decomposition). Molecular-level simulations are required to achieve a better understanding of these processes as well as to validate our current interpretation of the experimental cluster data. ${ }^{7,42-45,56}$ For cluster growth, we obtain very detailed, truly cluster-size resolved growth information over growth times of many hundred microseconds. This provides a unique experimental data set for future growth simulations. Qualitatively, we observe different growth regimes which are distinguished by the typical average size of the clusters and characteristic features in the size distribution (unimodal, multimodal, and disappearance of small clusters). Different simultaneous and competing mechanisms, such as growth from the vapor phase, cluster coagulation, and Ostwald ripening, are suggested as possible explanations.

\section{ACKNOWLEDGMENTS}

Wc are very grateful to Martina Lippe for her contribution to the deflector studies and to David Stapfer and Markus Steger from the LPC shops for their assistance in developing the experimental setup. Financial support was provided by the ETH Zürich and the Swiss National Science Foundation (SNF Project No. 200020_159205).

${ }^{1}$ K. Iland, J. Wölk, R. Strey, and D. Kashchiev, J. Chem. Phys. 127, 154506 (2007).

${ }^{2}$ S. Sinha, H. Laksmono, and B. E. Wyslouzil, Rev. Sci. Instrum. 79, 114101 (2008).

${ }^{3}$ S. Sinha, A. Bhabhe, H. Laksmono, J. Wölk, R. Strey, and B. E. Wyslouzil, J. Chem. Phys. 132, 064304 (2010).

${ }^{4}$ L. M. Feldmar, J. Wölk, and R. Strey, AIP Conf. Proc. 1527, 15 (2013).

${ }^{5} \mathrm{D}$. Ghosh, D. Bergmann, R. Schwering, J. Wölk, R. Strey, S. T'animura, and B. E. Wyslouzil, J. Chem. Phys. 132, 024307 (2010).

${ }^{6}$ K. Mullick, A. Bhabhe, A. Manka, J. Wölk, R. Strey, and B. E. Wyslouzil, J. Phys. Chcm. B 119, 9009 (2015).

${ }^{7}$ V. I. Kalikmanov, Nucleation Theory (Springer, Heidelberg, Netherlands, 2013).

${ }^{8}$ D. W. Oxtoby and R. Evans, J. Chem. Phys. 89, 7521 (1988).

${ }^{9}$ J. Wölk, J. Wedekind, R. Strey, and B. E. Wyslouzil, in Nucleation and Atmospheric Aerosols, edited by J. Smolik and C. O'Dowd (Institute of Chemical Process Fundamentals ASCR and Czech Aerosol Society, Prague, 2009), p. 589.

${ }^{10}$ B. Schläppi, J. H. Litman, J. J. Ferreiro, D. Stapfer, and R. Signorell, Phys. Chem. Chem. Phys. 17, 25761 (2015).

${ }^{11}$ J. J. Ferreiro, T. E. Gartmann, B. Schläppi, and R. Signorell, Z. Phys. Chem. 229, 1765 (2015).

${ }^{12}$ H. Laksmono, S. Tanimura, H. C. Allen, G. Wilemski, M. S. Zahniser, J. H. Shorter, D. D. Nelson, J. B. McManus, and B. E. Wyslouzil, Phys. Chem. Chem. Phys. 13, 5855 (2011).

${ }^{13}$ D. B. Atkinson and M. A. Smith, Rev. Sci. Instrum. 66, 4434 (1995).

${ }^{14}$ S. Lee, R. J. Hoobler, and S. R. Leone, Rev. Sci. Instrum. 71, 1816 (2000).

${ }^{15}$ T. Spangenberg, S. Köhler, B. Hansmann, U. Wachsmuth, B. Abel, and M. A. Smith, J. Phys. Chem. A 108, 7527 (2004).

${ }^{16}$ B. Hansmann and B. Abel, ChemPhysChem 8, 343 (2007).

${ }^{17}$ I. W. M. Sinith, Angew. Chem. 118, 2908 (2006).

${ }^{18}$ B. R. Rowe and J. B. Marquette, Int. J. Mass Spectrom. Ion Processes 80, 239 (1987).

${ }^{19}$ D. Chastaing, P. L. James, I. R. Sims, and I. W. M. Smith, Faraday Discuss. 109, 165 (1998).

${ }^{20}$ E. Vöhringer-Martinez, B. Hansmann, H. Hernandez, J. S. Francisco, J. Troe, and B. Abel, Science 315, 497 (2007).

${ }^{21}$ S. Hamon, S. D. Le Picard, A. Canosa, B. R. Rowe, and I. W. M. Smith, J. Chem. Phys. 112, 4506 (2000).

${ }^{22}$ L. Biennier, H. Sabbah, V. Chandrasekaran, S. J. Klippenstein, I. R. Sims, and B. R. Rowe, Astron. Astrophys. 532, A40 (2011).

${ }^{23}$ I. R. Sims, Nat. Chem. 5, 734 (2013).

${ }^{24}$ H. Sabbah, L. Biennier, S. J. Klippenstein, I. R. Sims, and B. R. Rowe, J. Phys. Chem. Lett. 1, 2962 (2010).

${ }^{25}$ I. R. Sims, J. L. Queffelec, A. Defrance, C. Rebrion-Rowe, D. Travers, P. Bocherel, B. R. Rowe, and I. W. M. Smith, J. Chem. Phys. 100, 4229 (1994).

${ }^{26}$ C. Abeysekera, L. N. Zack, G. B. Park, B. Joalland, J. M. Oldham, K. Prozument, N. M. Ariyasingha, I. R. Sims, R. W. Field, and A. G. Suits, J. Chem. Phys. 141, 214203 (2014).

${ }^{27}$ J. Daranlot, M. Jorfi, C. Xie, A. Bergeat, M. Costes, P. Caubet, D. Xie, H. Guo, P. Honvault, and K. M. Hickson, Science 334, 1538 (2011).

${ }^{28}$ B. Schläppi, J. J. Ferreiro, J. H. Litman, and R. Signorell, Int. J. Mass Spectrom. 372, 13 (2014).

${ }^{29}$ P. W. Forysinski, P. Zielke, D. Luckhaus, and R. Signorell, Phys. Chem. Chem. Phys. 12, 3121 (2010).

${ }^{30}$ B. L. Yoder, A. H. C. West, B. Schläppi, E. Chasovskikh, and R. Signorell, J. Chem. Phys. 138, 044202 (2013).

${ }^{31}$ C. Bobbert, S. Schütte, C. Steinbach, and U. Buck, Eur. Phys. J. D 19, 183 (2002).

${ }^{32}$ S. Schütte and U. Buck, Int. J. Mass Spectrom. 220, 183 (2002).

${ }^{33}$ B. L. Yoder, J. H. Litman, P. W. Forysinski, J. L. Corbett, and R. Signorell, J. Phys. Chem. Lett. 2, 2623 (2011).

${ }^{34}$ J. H. Litman, B. L. Yoder, B. Schläppi, and R. Signorell, Phys. Chem. Chem. Phys. 15, 940 (2013).

${ }^{35}$ L. Belau, K. R. Wilson, S. R. Leone, and M. Ahmed, J. Phys. Chem. A 111, 10075 (2007).

${ }^{36}$ F. Dong, S. Heinbuch, J. J. Rocca, and E. R. Bernstein, J. Chem. Phys. 124, 224319 (2006).

${ }^{37}$ J. Lengyel, A. Pysanenko, V. Poterya, J. Kočišek, and M. Fárník, Chem. Phys. Lett. 612, 256 (2014). 
${ }^{38}$ N. Daugey, P. Caubet, B. Retail, M. Costes, A. Bergeat, and G. Dorthe, Phys. Chem. Chem. Phys. 7, 2921 (2005).

${ }^{39}$ J. M. Oldham, C. Abeysekera, B. Joalland, L. N. Zack, K. Prozument, I. R. Sims, G. B. Park, R. W. Field, and A. G. Suits, J. Chem. Phys. 141, 154202 (2014).

${ }^{40}$ A. Bhabhe and B. E. Wyslouzil, J. Chem. Phys. 135, 244311 (2011).

${ }^{41}$ VDI-Gesellschaft, VDI-Wärmeatlas (Springer-Verlag, Berlin, Heidelberg, 2006).

${ }^{42}$ G. K. Schenter, S. M. Kathmann, and B. C. Garrett, Phys. Rev. I e.tt. 82, 3484 (1999).

${ }^{43}$ S. M. Kathmann, G. K. Schenter, B. C. Garrett, B. Chen, and J. I. Siepmann, J. Phys. Chem. C 113, 10354 (2009).

${ }^{44}$ D. Reguera and H. Reiss, J. Phys. Chem. B 108, 19831 (2004).

${ }^{45}$ D. Reguera and H. Reiss, Phys. Rev. Lett. 93, 165701 (2004).

${ }^{46}$ H. Lin and Y.-Y. Duan, J. Chem. Eng. Data 48, 1360 (2003).
${ }^{47}$ S. Tanimura, U. M. Dieregsweiler, and B. E. Wyslouzil, J. Chem. Phys. 133, 174305 (2010).

${ }^{48}$ S. Tanimura, H. Pathak, and B. E. Wyslouzil, J. Chem. Phys. 139, 174311 (2013).

${ }^{49}$ R. Strey and Y. Viisanen, J. Chem. Phys. 99, 4693 (1993).

${ }^{5 n}$ K. N. H. Looijmans, C. C. M. Luijten, and M. E. H. van Dongen, J. Chem. Phys. 103, 1714 (1995).

${ }^{51}$ H. Reiss, J. Chem. Phys. 18, 840 (1950).

${ }^{52}$ G. Wilemski, I. Phys. Chem. 91, 249? (1987).

${ }^{53}$ J. P. Garnier, P. Mirabel, and B. Migault, Chem. Phys. Lett. 115, 101 (1985).

${ }^{54}$ W. C. Hinds, Aerosol Technology: Properties, Behavior, and Measurement of Airborne Particles (John Wiley \& Sons Inc., New York, 1999).

55 J. H. Seinfeld and S. N. Pandis, Atmospheric Chemistry and Physics: From Air Pollution to Climate Change (John Wiley \& Sons Inc., New York, 2006).

${ }^{56}$ L. Inci and R. K. Bowles, J. Chem. Phys. 139, 214703 (2013). 